TITLE:

\title{
Why does substorm-associated auroral surge travel westward?
}

AUTHOR(S):

Ebihara, Y; Tanaka, T

CITATION:

Ebihara, Y ... [et al]. Why does substorm-associated auroral surge travel westward?. Plasma Physics and Controlled Fusion 2018, 60 (1): 14024.

ISSUE DATE:

2018-01

URL:

http://hdl.handle.net/2433/237229

\section{RIGHT:}

This is an author-created, un-copyedited version of an article accepted for publication in Plasma Physics and Controlled Fusion. The publisher is not responsible for any errors or omissions in this version of the manuscript or any version derived from it. The Version of Record is available online at https://orcid.org/0000-0002-2293-1557.; この論文は 出版社版でありません。引用の際には出版社版をご確認ご利用ください。; This is not the published version. Please cite only the published version. 


\title{
Why does substorm-associated auroral surge travel westward?
}

\author{
Y Ebihara ${ }^{1}$ and T Tanaka ${ }^{2}$ \\ 1. RISH, Kyoto University \\ 2. ICSWSE, Kyushu University \\ E-mail: ebihara@rish.kyoto-u.ac.jp
}

\begin{abstract}
A substorm is a long-standing unsolved issue in the solar-terrestrial physics. One of the big challenges is to explain reasonably the evolution of morphological structure of aurora associated with the substorm. Sudden appearance of bright aurora, and an auroral surge traveling westward (westward traveling surge, WTS) are a noticeable feature of the aurora during the substorm expansion phase. By using a global magnetohydrodynamics (MHD) simulation, we obtained the following results regarding the WTS. When the interplanetary magnetic field turns southward, a persistent dynamo appears in the cusp/mantle region, driving the two-cell magnetospheric convection. Then, the substorm growth phase begins. When magnetic reconnection takes place in the magnetotail, plasma is accelerated earthward in the plasma sheet, and accelerated toward the equatorial plane in the lobe. The second dynamo appears in the near-Earth region, which is closely associated with generation of the FACs on the nightside. When the FACs reached the ionosphere, the aurora becomes bright, and the expansion phase onset begins. In the ionosphere, the conductivity is intensified in the bright aurora due to precipitation of accelerated electrons. The conductivity gradient gives rise to overflow of the Hall current, which acts as the third dynamo. The overflow results in accumulation of space charge, which causes divergent electric field. The divergent electric field generates a thin, structured upward FAC adjacent to the bright aurora. The opposite process takes place on the opposite side of the bright aurora. In short, the upward FAC increases (appearance of aurora) at the leading edge of the surge, and decreases (disappearance of aurora) at the trailing edge of the surge. By repeating these processes, the surge seems to travel westward.
\end{abstract}




\section{Introduction}

Aurora is an impressive, brilliant phenomenon occurring as a final consequence of the dynamical processes in the solar wind-magnetosphere-ionosphere coupling system. Most of the aurora can be seen on the auroral oval which is circularly located around the magnetic pole with its latitudes about $67^{\circ}$ at midnight and about $76^{\circ}$ at noon. After the interplanetary magnetic field (IMF) turns southward, a sequence of auroral morphological changes known as the substorm starts. During the growth phase of the substorm, faint auroral arcs, usually aligned with the north-south (N-S) or east-west (E-W) directions, move from the polar region to equatorward. At this time, the tail magnetic field lines are stretched due to the corresponding dynamics in the magnetotail region. Occasionally, a sudden brightening of one of the auroral arcs occurs near midnight, followed by expansion of the bright aurora, including a bulge and a surge. This is called an expansion phase. The near-Earth neutral line in the tail region is believed to be closely associated with substorm expansion . The bright aurora expands primarily in the poleward and westward directions. The expanded aurora gradually returns to the original state. This cycle of the evolution of the aurora is called a substorm (Akasofu, 1964).

The bright aurorae are usually caused by precipitating electrons due to parallel electric field at low altitude. The upward electric field, which accelerates electrons downward, is known to be embedded in the upward field-aligned current (FAC) (Lyons, Evans and Lundin, 1979; Weimer et al., 1987; Elphic et al., 1998). In conjunction with significant ionization of the ionosphere, a pair of the upward and downward FACs give rise to intensify the ionospheric current, known as an auroral electrojet (Boström, 1964; Atkinson, 1967; Armstrong, Akasofu and Rostoker, 1975; Kamide and Akasofu, 1976). In a substorm study, of particular interest is the generation of the upward FAC that consisute a part of the current wedge (CW) (McPherron, Russell and Aubry, 1973). Originally, the CW has been regarded as a diversion of the cross-tail current that flows westward. The FACs flow along a field line from the equatorial plane to the ionosphere on the dawnside, and out of the ionosphere on the duskside. Recently, the $\mathrm{CW}$ has also been considered as a result of a superposition of some current loops including FACs generated by flow shear in the plasma sheet (Kepko et al., 2015). However, important aspects of the FAC are not necessarily considered in the $\mathrm{CW}$ model. One of the important aspects of the FAC is that it occurs to transmit motion from the magnetosphere to the ionosphere. The other aspect is the association of dynamo that convert energy so as to drive the ionospheric convection. The associated dynamo region in the CW had been a problematic (Akasofu, 2003; Lui and Kamide, 2003). 
The global MHD simulation is now capable of reproducing major auroral features that emerge from quiet time to the substorm expansion phase. It reproduces the auroral structures (upward FACs) that resemble the N-S and E-W arcs moving equatorward during the growth phase. It is also capable to reproduce the upward FAC that is similar to the initial brightening and the WTS. The magnetospheric processes and resulting ionospheric signatures are confirmed to be consistent with observations (Tanaka 2015; Ebihara \& Tanaka 2015ab; Ebihara \& Tanaka 2016; Tanaka et al. 2017).

In the convection reproduced by the recent MHD simulation, it has become clear how the FAC corresponds to the convection shear, and how the energy conversion exciting the dynamo occurs. These results have shown that a persistent, strong dynamo exists in the cusp/mantle region resulting in the Region 1 FACs (Tanaka, 1995; Siscoe et al., 2000). For the Region 1 dynamo, the following explanation is suggested (Tanaka, 2007; Tanaka et al., 2016). As a consequence of the dayside magnetopause reconnection, the plasma originating in the solar wind is accelerated. The plasma is then decelerated inside the magnetosheath. The kinetic energy is converted to the electromagnetic energy. The electromagnetic energy is transported into the magnetosphere so as to increase the plasma pressure in the cusp, that is, an increase in the thermal energy. The thermal energy is converted to the electromagnetic energy as the plasma moves tailward in the cusp/mantle region. It was shown that energy conversion is also dominant even during the substorm. An onset dynamo is found in the near-Earth region, which is closely associated with high-pressure region generated by the injection (Birn \& Hesse 2005; Tanaka 2015; Ebihara \& Tanaka 2015a).

In addition to the magnetospheric origin, the ionosphere is suggested to play a role in the formation of the upward FACs associated with the westward traveling surge (WTS). A localized upward FAC is suggested to form on the western edge of the surge where the conductivity is too high to maintain the current continuity within the ionosphere (Baumjohann et al. 1981; Inhester et al. 1981; Opgenoorth et al. 1983). The overflow of the ionospheric Hall current is found to be responsible for the formation of WTS (Kan, Williams and Akasofu, 1984; Kan and Kamide, 1985). A feedback instability is also proposed between the magnetosphere and the ionosphere (Rothwell et al. 1984; Rothwell et al. 1988). Using the global MHD simulation, Ebihara and Tanaka (2015b) 
showed the formation and evolution of the WTS in terms of the partial blockage of the Hall current and newly generated upward FACs. The generation of the upward FACs was described, but the reason why the surge travels westward was not explicitly explained. The aim of this paper is to explain the possible reason why the surge travels westward by re-analysing the global MHD simulation result.

\section{Simulation method}

We used the global MHD simulation that couples the magnetosphere and the ionosphere (Tanaka 1995; Tanaka et al. 2010; Tanaka 2015). In the simulation, the magnetospheric dynamics is reproduced from the MHD equation. In addition, a small dissipation term is included in the MHD equation to realize migration of magnetic field lines on the magnetopause. This process enables the development of MHD system that even accompanies the change of magnetic topology. The inner and outer boundaries of the magnetospheric domain are set at spheres with radius of $3 \mathrm{Re}$ and $300 \mathrm{Re}$, respectively. A unique grid system is employed for the discretization of the magnetospheric domain. This grid system is necessary to avoid apparent singularities. First, a sphere is divided into 12 pentagons. Each pentagon is divided into 5 triangles. We call this Level 1 gridding. We repeated the division 7 times, which is called Level 7. 380 triangular prisms were stacked outward from the inner boundary. The total number of the grid points was 46,572,278. A finite volume TVD scheme was used to solve the MHD equations.

By mapping plasma pressure, temperature and FACs from the inner boundary of the magnetospheric domain $(3 \mathrm{Re})$ to the ionosphere $(1 \mathrm{Re})$, we calculated the ionospheric conductivity in the ionosphere at 1 Re. Under the given ionospheric conductivity and FACs mapped from $3 \mathrm{Re}$, we solved an elliptic partial differential equation on a sphere at $1 \mathrm{Re}$ to derive the ionospheric electric potential. This potential is equivalent to ionospheric convection. The electric potential was mapped back to the inner boundary of the magnetospheric domain. The plasma velocity corresponding to the electric field was imposed to the MHD boundary condition at $3 \mathrm{Re}$.

The coordinate system is essentially the same as the Solar Magnetospheric coordinates. The $X$ axis points toward the Sun, and the $Z$-axis is anti-parallel to Earth's dipole moment. The $Y$-axis is defined as the cross-product of the $Z$ - and $X$-axes. In order to obtain a quasi-steady state 
magnetosphere, we gave the solar wind a density of $5.0 \mathrm{~cm}^{-3}$, a solar wind speed of $372 \mathrm{~km} / \mathrm{s}$, the $Y$-component of the interplanetary magnetic field (IMF $B_{y}$ ) of $-2.5 \mathrm{nT}$, and IMF $\mathrm{B}_{\mathrm{z}}$ of $4.3 \mathrm{nT}$ (northward). We then changed IMF $B_{z}$ to -3.0 nT (southward). See Ebihara and Tanaka (2015b) for the simulation in detail.

\section{Results}

First of all, we show overall evolution of the auroral substorm simulated by the global MHD simulation. Figure 1 shows evolution of the calculated FACs at the ionosphere. We defined $T=0$ as the moment when the southward IMF reached $X=40$ Re. The MHD simulation cannot solve the electric field parallel to the magnetic field. Instead, the upward FAC is used to be a proxy of the bright aurora because the upward electric field is known to be embedded in the upward FACs. Hereinafter, we call the upward FAC an aurora for the sake of simplicity.

18

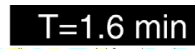

$-\mathrm{NS}$ aurora

80

70

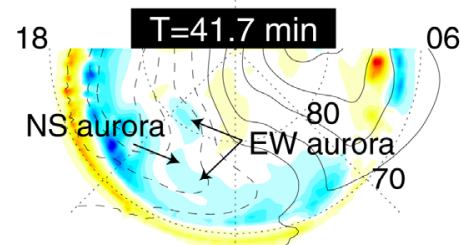

18

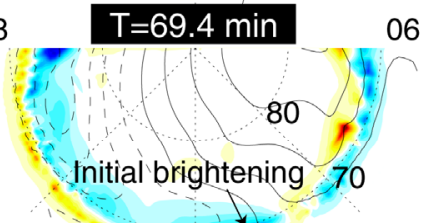

, 18

$\mathrm{T}=73.0 \mathrm{~min}$

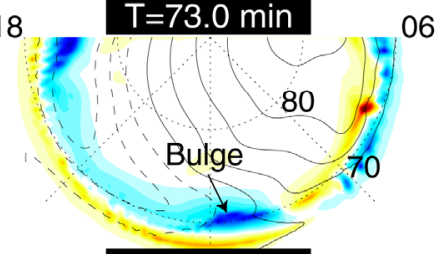

18
$06 \quad 18$

$T=31.5 \mathrm{~min}$

-EW aurora

- NS aurora

1) $\quad 70$

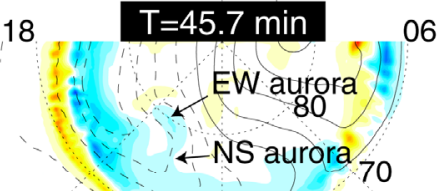

18

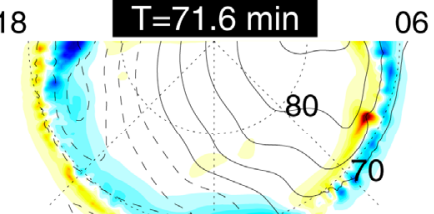

18

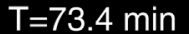

80

Surge

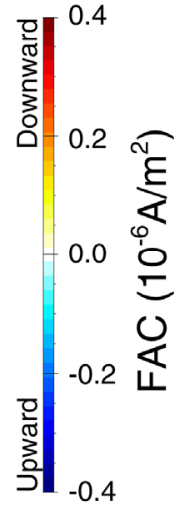

Surge

80

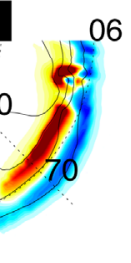


Figure 1. Evolution of auroral substorm from quiet time to expansion phase. Each panel shows calculated field-aligned currents (FACs) in the ionosphere. Blue color and red color indicate the upward and downward FACs, respectively. The contour line indicates the ionospheric electric potential at an interval of $5 \mathrm{kV}$. The solid and dashed lines indicate the positive and negative potentials, respectively. The Sun is to the top. The lower boundary is located at 65 magnetic latitudes (MLATs).

At $T=1.6 \mathrm{~min}$, the magnetosphere is exposed to the northward IMF. The geomagnetic activity is very low. A thin auroral form is evident on the nightside. The auroral form results primarily from a Rayleigh Taylor-like instability due to an electromagnetic interaction between the magnetosphere and the ionosphere (Ebihara and Tanaka, 2016). A non-uniform distribution of the plasma pressure generates meso-scale FACs in the lobe. When the meso-scale FACs arrives at the ionosphere, an additional electric field is generated to maintain the current continuity in the ionosphere. The electric field propagates to the magnetosphere so as to redistribute the magnetospheric plasmas, and to enlarge the distortion of the plasma pressure distribution. When the magnetosphere is exposed to the southward IMF, the magnetospheric convection is stimulated. Two-cell convection pattern as indicated by black contour lines appears in the ionosphere; one is on the duskside, and the other is on the dawnside. The substorm growth phase is initiated. The thin auroral form still reside at $T=31.5 \mathrm{~min}$, but the structure is slightly deformed. The thin auroral from moves equatorward as time proceeds. Auroral forms aligned with north-south direction and east-west direction appear. The morphological structure and evolution of the auroral form resemble the observed ones (Nishimura et al., 2010). Finally, it merges into the pre-existing main auroral oval. The equatorward movement of the auroral form corresponds to the movement of the structured plasma pressure distribution toward the equatorial plane in the lobe. An eastwest aligned auroral arc is also suggested to appear due to shear motion of plasma near the boundary between the lobe and the plasma sheet (Tanaka, 2015). At $T \sim 55 \mathrm{~min}$, a near-Earth neutral line (NENL) starts to form at $X \sim-42 \mathrm{Re}$. The magnetosphere starts to be reconfigured in a 3-dimensional manner. Earthward and anti-earthward fast flows appear in the equatorial plane, which result in flow vorticity in the equatorial plane. In the lobe region, plasma starts to move toward the equatorial plane, resulting in flow vorticity. The flow vorticities are related to the FACs that manifest the expansion phase. At $T=69.4 \mathrm{~min}$, a thin, bright aurora starts to appear, which is elongated to the azimuthal direction. This may correspond to the beginning of the substorm expansion phase. The location of the brightening belongs to the duskside convection cell. The thickness becomes large, which may correspond to a bulge. Finally, a thin, bright aurora 
is detached from the bulge, which is called a surge. The surge travels westward as time proceeds. This is similar to the WTS.

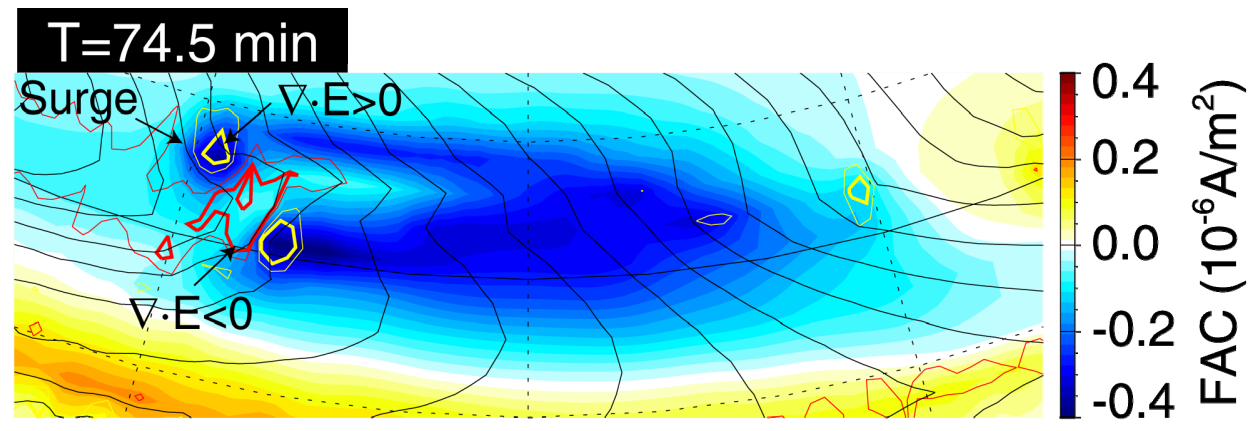

Figure 2. Close-up view of the FACs near the surge at $T=74.5 \mathrm{~min}$. The color code is the same as Figure 1. The yellow and red contour lines indicate the region where $\nabla \cdot \boldsymbol{E}$ is positive $\left(0.3 \times 10^{-6} \mathrm{~V} / \mathrm{m}^{2}\right.$ for thick line and $0.15 \times 10^{-6} \mathrm{~V} / \mathrm{m}^{2}$ for thin line $)$ and $\boldsymbol{\nabla} \cdot \boldsymbol{E}$ is negative $\left(-0.3 \times 10^{-6}\right.$ $\mathrm{V} / \mathrm{m}^{2}$ for thick line and $-0.15 \times 10^{-6} \mathrm{~V} / \mathrm{m}^{2}$ for thin line), respectively. $\boldsymbol{E}$ is the ionospheric electric field. The contour line indicates the ionospheric electric potential at interval of $1 \mathrm{kV}$.

There is an argument regarding the polarity of $\nabla \cdot \mathbf{E}$ in the surge. On the basis of observations, positive $\nabla \cdot \mathbf{E}$ (Weimer et al., 1994) and negative $\nabla \cdot \mathbf{E}$ (Marklund et al., 1998, 2012) are suggested. Figure 2 is a close-up view of the FACs near surge at $T=74.6 \mathrm{~min}$. The contour line indicates the ionospheric electric potential. The electric potential is high on the dawnside, and low on the duskside. As identified from the electric potential lines, there is a primary electric field pointing westward approximately. $\nabla \cdot \mathbf{E}$ is negative westward of the surge, which is probably associated with a closure of the Region 1-sense FACs, namely the primary electric field. The equatorward component of the electric field also arises in the surge, which is probably associated with the secondary electric field, known as Cowling channel, or blockage of the Hall current (Baumjohann et al., 1981; Inhester et al., 1981; Opgenoorth et al., 1983; Kan and Kamide, 1985). As a consequence, the potential pattern is highly skewed, which is similar to that drawn by Weimer et al. (1994). Most of the Hall current is connected to the FACs, but a small amount of the Hall current is connected with the divergent Pedersen current (Kan et al. 1984; Kan \& Kamide 1985; Ebihara \& Tanaka, 2015b). The positive $\nabla \cdot \mathbf{E}$ means that positive space charge is accumulated. 
Figure 3 shows $\nabla \cdot \mathbf{E}$ at geocentric distances at 3.2 Re and 4.0 Re. At 3.2 Re, small-scale, divergent electric field $(\nabla \cdot \mathbf{E}>0)$ appears above the surge along a field line. Noticeably, the divergent electric field is embedded in the large-scale, convergent electric field $(\nabla \cdot \mathbf{E}<0)$. At $4.0 \mathrm{Re}$, the small-scale divergent electric field is absent. The small-scale upward FACs associated with the surge is connected to the divergent inertial current at low altitude, so that they are absent at 4.0 Re (not shown). This indicates that the small-scale divergent electric field associated with the surge is embedded in the large-scale convergent electric field (and hence, large-scale upward FACs) at low altitude.

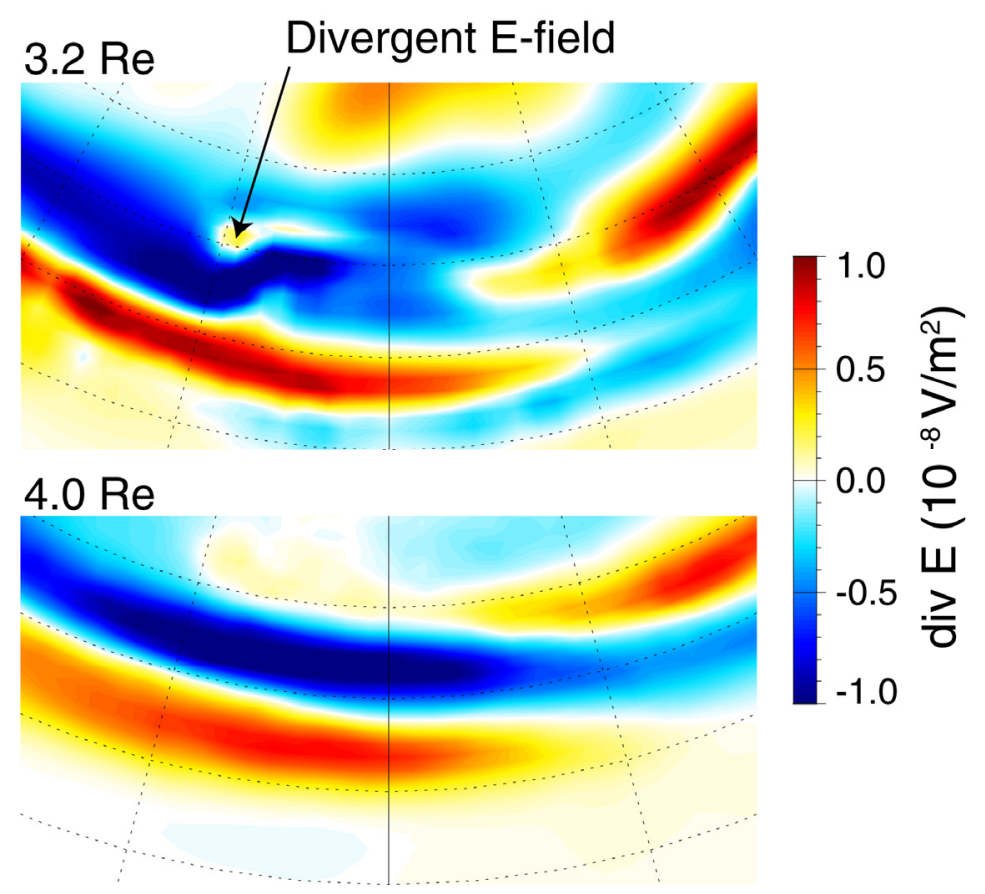

Figure 3. $\nabla \cdot \mathbf{E}$ on a spherical surface at geocentric distances at $3.2 \mathrm{Re}$ and $4.0 \mathrm{Re}$ in the magnetosphere over the serge at $T=74.5 \mathrm{~min}$. The divergent electric field associated with the surge is confined at low altitude.

Figure 4 shows $\mathbf{J} \cdot \mathbf{E}$ at 3.2 Re. $\mathbf{J} \cdot \mathbf{E}$ is negative above the leading edge of the surge as indicated by the arrow. The negative $\mathbf{J} \cdot \mathbf{E}$ region corresponds to the positive $\nabla \cdot \mathbf{E}$ region as shown in Figure 3. We will discuss the relationship between $\nabla \cdot \mathbf{E}$ and $\mathbf{J} \cdot \mathbf{E}$ later. 


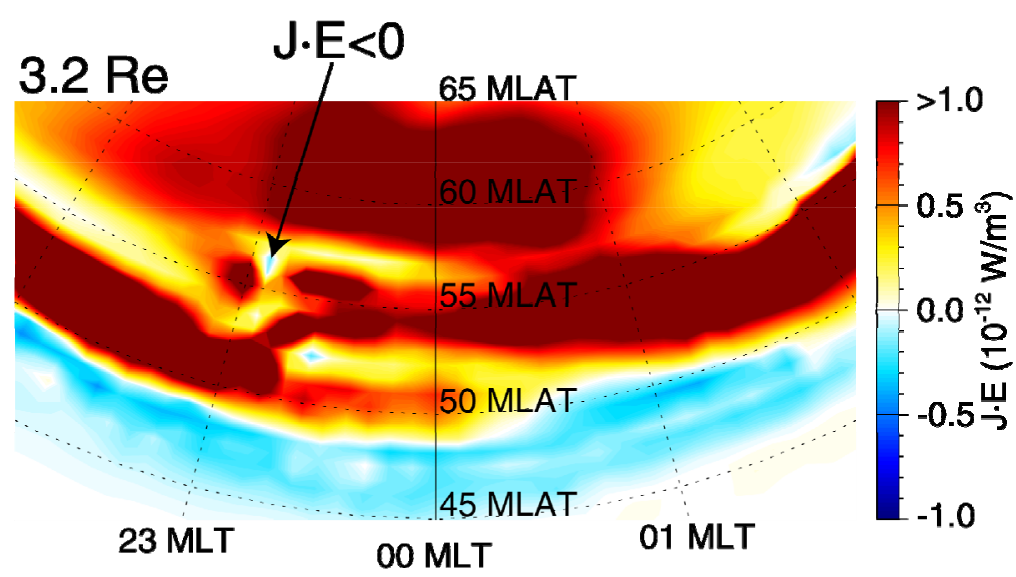

Figure 4. $\mathbf{J} \cdot \mathbf{E}$ on a spherical surface at geocentric distance at 3.2 Re in the magnetosphere at $T$ $=74.5 \mathrm{~min}$.

The small-scale, divergent electric field is embedded in the large-scale, convergent electric field at low-altitude magnetosphere. The convergent electric field is associated with the large-scale upward FACs, known as a Region 1-sence FAC. We present an integral curve of the current density vector to show the origin of the large-scale upward FAC. Figure 5 shows the integral curves of the current density vector from the surge. The color code on the integral curve indicates the value of $\mathbf{J} \cdot \mathbf{E}$. The current line passes through two major dynamo regions where $\mathbf{J} \cdot \mathbf{E}<0$. First one is the near-Earth dynamo (Birn \& Hesse 2005; Tanaka 2015; Ebihara \& Tanaka 2015a), and second one is the cusp/mantle dynamo (Tanaka, 1995; Siscoe et al., 2000). In the global MHD simulation, the formation of the near-Earth neutral line initiates the reconfiguration of the magnetosphere. The plasma is accelerated Earthward in the equatorial plane, and is also accelerated toward the equatorial plane in the lobe. Thus, the reconfiguration takes place in a 3dimensional manner. As a consequence, a pair of flow vortices appears. A pair of FACs are generated due to the flow vorticites; upward FAC on the duskside and downward FAC on the dawnside. The global MHD simulation result shows that the third dynamo may exist in the ionosphere and low-altitude magnetosphere due to the charge separation associated with the overflow of the ionospheric Hall current as shown in Figure 4. 


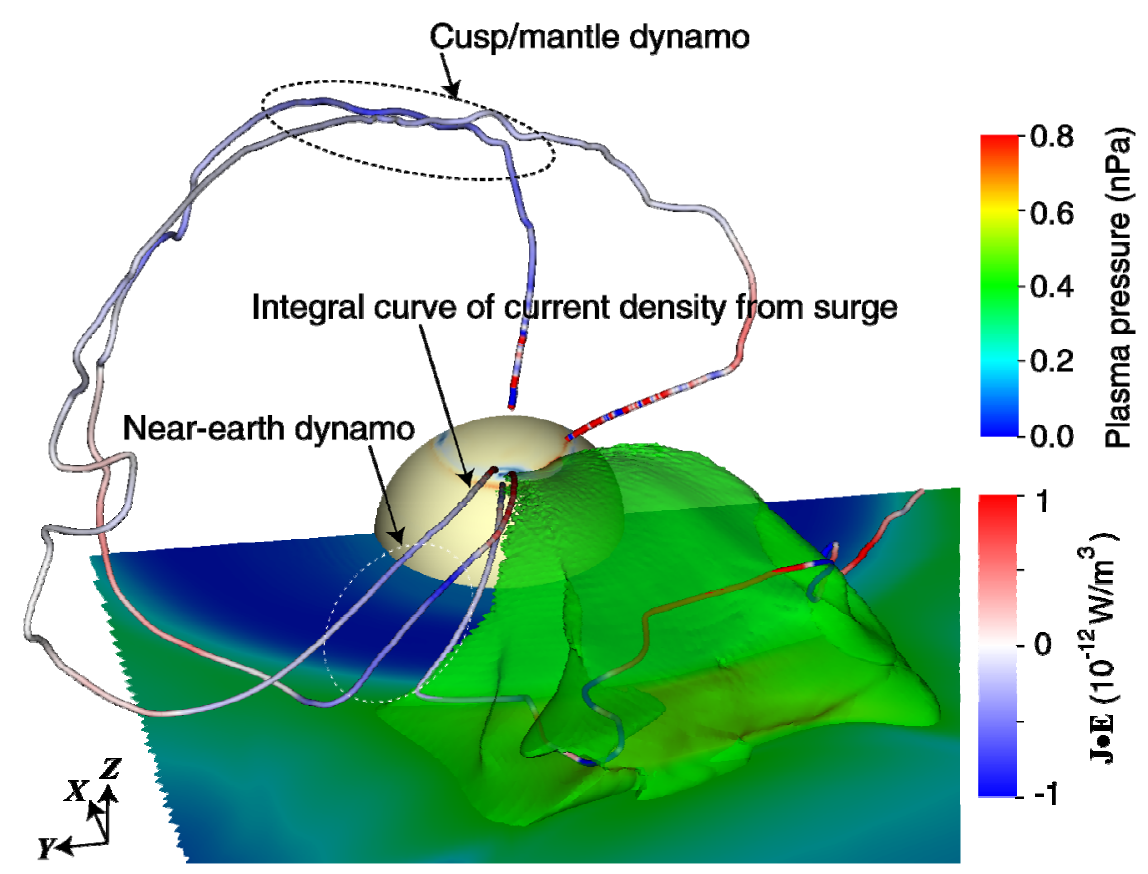

Figure 5. Perspective view of the magnetosphere at $T=74.5 \mathrm{~min}$. The greenish surface indicates that plasma pressure is $0.4 \mathrm{nPa}$. Integral curves of the current density vector are drawn. The color code on the integral curves indicates the value of $\mathbf{J} \cdot \mathbf{E}$. There are two dynamo regions associated with the substorm expansion; cusp/mantle dynamo and near-Earth dynamo. (After Ebihara and Tanaka, 2015b)

In the simulation, the ionospheric conductivity is enhanced where the upward FAC is high and/or the plasma pressure is high. Apart from reality, we assumed that the downward FAC also results in the enhancement of the ionospheric conductivity as much as the upward FAC does. Figure 6 shows an example of the FACs during the expansion phase. A surge-like structure of the downward FAC travels eastward on the dawnside. This clearly indicates the importance of the conductivity gradient in the ionosphere in the formation of the traveling surge. In reality, the downward FAC does not cause bright aurora and enhancement of ionospheric conductivity, so that the eastward surge is invisible. That is why only the surge traveling westward is visible. 


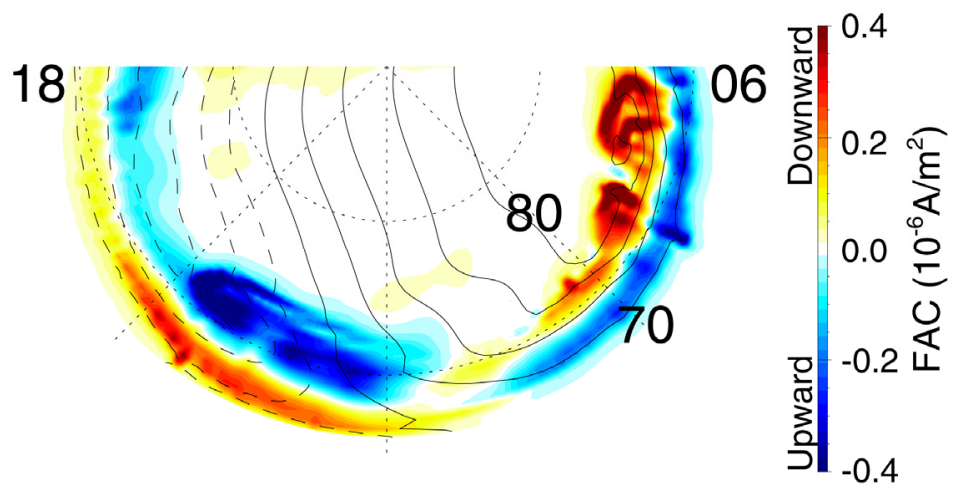

Figure 6. FACs at $T=79.2$ min in case that the downward FAC also results in the enhancement of the ionospheric conductivity as much as the upward FAC does. The Sun is to the top. A surgelike structure of downward FAC is found to travel eastward on the dawnside.

\section{Discussion and summary}

To understand the simulation result, let us consider an elongated bright auroral region where the ionospheric conductivity is higher than ambient as shown in Figure 7. For the sake of simplicity, the ionosphere is considered to be a thin layer. Because of the presence of the primary electric field, the ionospheric Hall current flows from the right to the left. The magnitude of the ionospheric Hall current is larger in the surge than ambient because the ionospheric conductivity is higher than ambient due to precipitating electrons. In the simulation, the ionospheric conductivity increases in accordance with the upward FACs and the plasma pressure mapped from the inner boundary of the magnetospheric domain. The Hall current overflows near the lefthand side (leading edge) of the surge, and the Hall current lacks near the right-hand side (trailing edge) of the surge. Most of the Hall current is connected to the FACs, but a small portion of the Hall current is connected to the Pedersen current. The secondary electric field has positive divergence, which means positive space charge. Detailed electrodynamics near the surge is also discussed by Haerendel $(2008,2009)$ and Fujii et al. (2011). Shear in the counterclockwise (clockwise) direction is expected to appear above the leading edge (trailing edge) of the surge, and to propagate upward. The upward Poynting flux is also expected because the ionosphere acts as a current generator, or a transformer (Haerendel, 2008). 


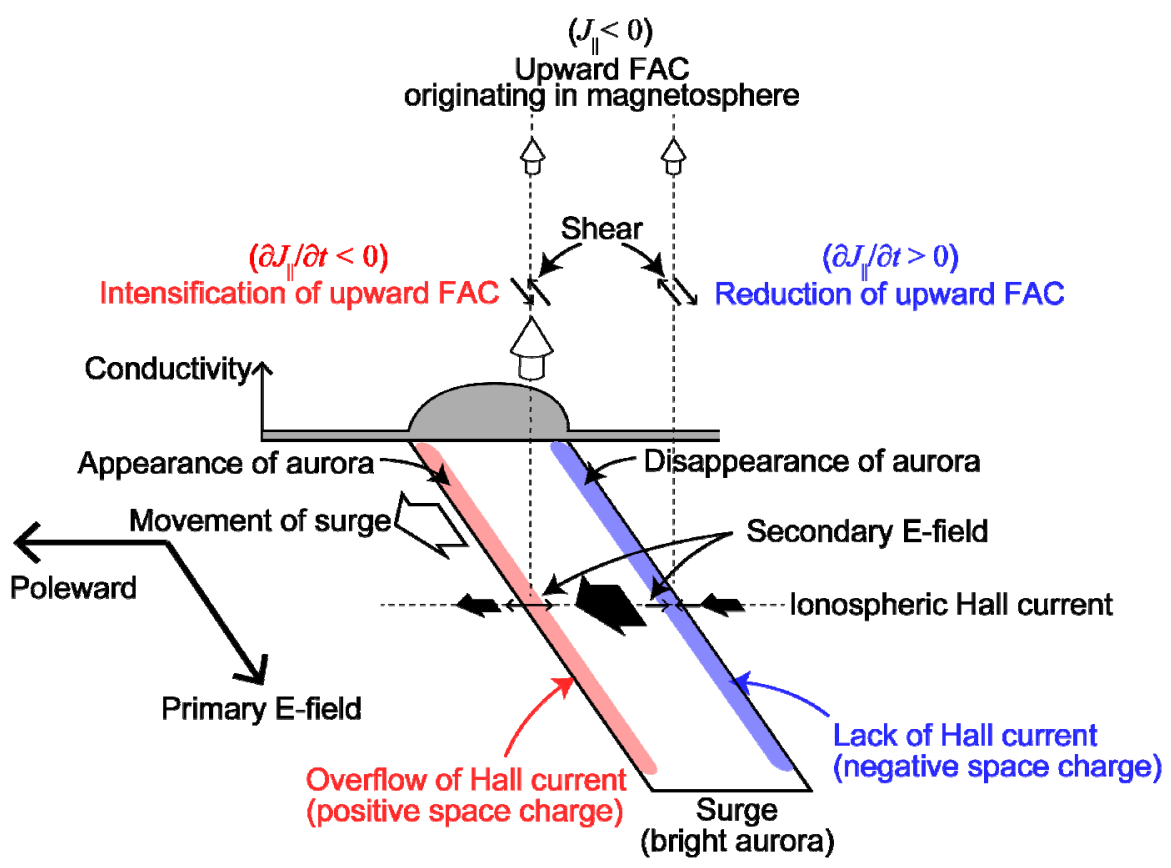

Figure 7. Schematic drawing of the surge, the ionospheric Hall current, and the convection electric field. The Northern Hemisphere is assumed, in which the magnetic field looks downward. Note that the poleward direction indicated by the arrow is arbitrary.

Next, let us consider the generation of FACs. The generation of the FACs is given by the following equation from Faraday's and Ampère's laws.

$$
\frac{\partial J_{\|}}{\partial t}=-\frac{1}{\mu_{0}}[\nabla \times \nabla \times \mathbf{E}]_{\|}
$$

where $J_{\|}$is the FAC ( $\equiv \mathbf{J} \cdot \mathbf{B} / B$, where $\mathbf{B}$ is the magnetic field), and $\mathbf{E}$ is the electric field. Eq. (1) yields (Song and Lysak, 2001)

$$
\begin{aligned}
\frac{\partial J_{\|}}{\partial t} & =-\frac{1}{\mu_{0}}\left[\nabla_{\|}\left(\nabla_{\perp} \cdot \mathbf{E}\right)-\nabla_{\perp}^{2} \mathbf{E}_{\|}\right] \\
& \simeq \frac{1}{\mu_{0}} \mathbf{B} \cdot \nabla \Omega_{\|},
\end{aligned}
$$

where $\Omega_{\|}$is the parallel vorticity $(\equiv(\nabla \times \mathbf{V}) \cdot \mathbf{B} / B)$. In the MHD equation, $\mathbf{E}_{\|}$vanishes. Here, we assumed that $\mathbf{B} \cdot \mathbf{\Omega}_{\|} \gg \mu_{0} \mathbf{V} \cdot \mathbf{J}_{\perp}$, where $\mathbf{V}$ is the plasma flow velocity. Eq. (2) means that the rate of change in FAC depends on changes in $\nabla \cdot \mathbf{E}$, or parallel vorticity along a field line. At the 
leading edge of the surge, the divergent electric field is expected to appear, which corresponds to the negative vorticity (counterclockwise flow when one views along a field line). The divergent electric field (counterclockwise flow vorticity) imposed on the ionosphere would propagate upward along a field line. The magnitude of the negative vorticity probably decreases with altitude because the negative vorticity is imposed at low altitude. In this case, $\mathbf{B} \cdot \nabla \Omega_{\|}$is negative above the leading edge of the surge along a field line, giving rise to the generation of the upward FAC $\left(\partial J_{\|} / \partial t<0\right)$.

In the simulation, the upward FACs result in the intensification of the ionospheric conductivity. The additionally intensified upward FACs enhance the ionospheric conductivity giving rise to the overflow of the Hall current adjacent to the surge. Thus, the bright aurora propagates toward the left-hand side in Figure 7. The opposite processes take place at the trailing edge of the surge. A convergent electric field is expected to appear at the trailing edge of the surge, and the upward FACs are suppressed, according to Eq.(2). Thus, the bright aurora disappears at the trailing edge of the surge. By repeating these processes, the surge moves to the left. From the above consideration, the surge is expected to travel along the equipotential line (direction of the Hall current). As shown in Figure 2, the brightening (intensification of the upward FACs) occurs in the duskside convection cell. The direction of the Hall current is primarily westward in the vicinity of the surge. This is probably one of the reasons why the surge travels westward. The strong gradient of the ionospheric conductivity occurs in association with the upward FACs. A surgelike structure of the downward FACs traveling eastward is absent as shown in Figure 6.

The global MHD simulation is capable of reproducing the upward FAC structure that is similar to WTS. In the global MHD simulation, the WTS appears due to the following processes. (1) The brightness of auroral (magnitude of the upward FACs) starts to increase due to the formation of the near-Earth dynamo, which is triggered by the formation of the near-Earth neutral line. (2) The ionospheric Hall current overflows near the bright aurora (high conductivity region). (3) The overflow of the ionospheric Hall current results in the divergent electric field, generating the upward FAC adjacent to the bright aurora (surge). (4) The space charge is lack on the other side of the bright aurora. The opposite process takes place to reduce the upward FACs. (5) Once the surge is generated, the upward FAC is generated at the leading edge of the surge, and becomes weak at the trailing edge of the surge. (6) By repeating these processes, the surge travels westward. 
At low altitude $(<10,000 \mathrm{~km}$ altitude), parallel electric field becomes important to accelerate electrons downward, so as to generate bright auroral structures. The bright auroral structures are suggested to have a clockwise rotation as viewed from above in the Northern Hemisphere (Oguti, 1974). If motion of the auroral structures follows the $E \times B$ drift, the clockwise rotation will correspond to the convergence of the electric field, that is, excess of negative charge. This seems to be inconsistent with the simulation result in which the divergence of the electric field is expected in the leading edge of the surge. The global MHD simulation cannot deal with the parallel electric field. Of course, we cannot rule out the possibility that the WTS is a direct manifestation of energy conversion in the nightside magnetosphere, such as a high-beta plasma layer (Haerendel 2008, 2009). More detailed simulation including electron kinetics, as well as more detailed analysis of data from satellites or radar are probably needed to confirm the existence of the divergence of the electric field. The divergence of the electric field at the leading edge of the surge is an issue to be resolved in the future study.

\section{Acknowledgements.}

The computer simulation was performed on the A-KDK computer system at the Research Institute for Sustainable Humanosphere (RISH), Kyoto University. This study was supported by JSPS KAKENHI Grant Numbers 15H03732, and 15H05815.

\section{References}

Akasofu, S.-I. (1964) 'The development of the auroral substorm', Planetary and Space Science, 12(4), pp. 273-282. doi: 10.1016/0032-0633(64)90151-5.

Akasofu, S.-I. (2003) 'A source of auroral electrons and the magnetospheric substorm current systems', Journal of Geophysical Research, 108(A4), 8006. doi: 10.1029/2002JA009547.

Armstrong, J. C., Akasofu, S.-I. and Rostoker, G. (1975) 'A comparison of satellite observations of Birkeland currents with ground observations of visible aurora and ionospheric currents', Journal of Geophysical Research, 80(4), pp. 575-586. doi: 10.1029/JA080i004p00575.

Atkinson, G. (1967) 'An approximate flow equation for geomagnetic flux tubes and its 
application to polar substorms', Journal of Geophysical Research, 72(21), pp. 5373-5382. doi: 10.1029/JZ072i021p05373.

Baumjohann, W., Pelunen, R. J., Opgenoorth, H. J. and Nielsen, E. (1981) 'Joint twodimensional observations of ground magnetic and ionospheric electric fields associated with auroral zone currents: Current systems associated with local auroral break-ups', Planetary and Space Science, 29(4), pp. 431-447. doi: 10.1016/0032-0633(81)90087-8.

Birn, J. and Hesse, M. (2005) 'Energy release and conversion by reconnection in the magnetotail', Annales Geophysicae, 23(10), pp. 3365-3373. doi: 10.5194/angeo-23-33652005.

Birn, J. and Hesse, M. (2005) 'Energy release and conversion by reconnection in the magnetotail', Annales Geophysicae, 23(10), pp. 3365-3373. doi: 10.5194/angeo-23-33652005.

Boström, R. (1964) 'A model of the auroral electrojets', Journal of Geophysical Research, 69(23), p. 4983. doi: 10.1029/JZ069i023p04983.

Ebihara, Y. and Tanaka, T. (2015) 'Substorm simulation: Insight into the mechanisms of initial brightening', Journal of Geophysical Research: Space Physics, 120(9), pp. 7270-7288. doi: 10.1002/2015JA021516.

Ebihara, Y. and Tanaka, T. (2016) 'Substorm simulation: Quiet and N-S arcs preceding auroral breakup', Journal of Geophysical Research: Space Physics, 121(2), pp. 1201-1218. doi: 10.1002/2015JA021831.

Elphic, R. C., Bonnell, J. W., Strangeway, R. J., Kepko, L., Ergun, R. E., McFadden, J. P., Carlson, C. W., Peria, W., Cattell, C. A., Klumpar, D., Shelley, E., Peterson, W., Moebius, E., Kistler, L. and Pfaff, R. (1998) 'The auroral current circuit and field-aligned currents observed by FAST', Geophysical Research Letters, 25(12), pp. 2033-2036. doi: 10.1029/98GL01158.

Fujii, R., Amm, O., Yoshikawa, A., Ieda, A. and Vanhamäki, H. (2011) 'Reformulation and energy flow of the Cowling channel', Journal of Geophysical Research, 116(A2), p. A02305. doi: 10.1029/2010JA015989.

Haerendel, G. (2008) 'Auroral arcs as current transformers', Journal of Geophysical Research: Space Physics, 113, pp. 1-16. doi: 10.1029/2007JA012947. 
Haerendel, G. (2009) 'Poleward arcs of the auroral oval during substorms and the inner edge of the plasma sheet', Journal of Geophysical Research: Space Physics, 114(A6). doi: 10.1029/2009JA014138.

Hones, E. W., Asbridge, J. R., Bame, S. J. and Singer, S. (1973) 'Substorm variations of the magnetotail plasma sheet from $\mathrm{X} \mathrm{SM} \approx-6 \mathrm{R}_{\mathrm{E}}$ to $\mathrm{X} \mathrm{SM} \approx-60 \mathrm{R}_{\mathrm{E}}$, Journal of Geophysical Research, 78(1), pp. 109-132. doi: 10.1029/JA078i001p00109.

Inhester, B., Baumjohann, W., Greenwald, R. A. and Nielsen, E. (1981) 'Joint two-dimensional observations of ground magnetic and ionospheric electric fields associated with auroral zone currents. III - Auroral zone currents during the passage of a westward travelling surge', $J$. Geophys., 49, pp. 155-162.

Kamide, Y. and Akasofu, S. I. (1976) 'The Auroral electrojet and field-aligned current', Planetary and Space Science, 24(3), pp. 203-213. doi: 10.1016/0032-0633(76)90017-9.

Kan, J. R. and Kamide, Y. (1985) 'Electrodynamics of the westward traveling surge', 90, pp. 7615-7619. doi: 10.1029/JA090iA08p07615.

Kan, J. R., Williams, R. L. and Akasofu, S.-I. (1984) 'A mechanism for the westward traveling surge during substorms', Journal of Geophysical Research, 89(A4), p. 2211. doi: 10.1029/JA089iA04p02211.

Kepko, L., McPherron, R. L., Amm, O., Apatenkov, S., Baumjohann, W., Birn, J., Lester, M., Nakamura, R., Pulkkinen, T. I. and Sergeev, V. (2015) 'Substorm current wedge revisited', Space Science Reviews. The Author(s), 190(1-4), pp. 1-46. doi: 10.1007/s11214-014-01249.

Lui, A. T. Y. and Kamide, Y. (2003) 'A fresh perspective of the substorm current system and its dynamo', Geophysical Research Letters, 30(18), 1958. doi: 10.1029/2003GL017835.

Lyons, L. R., Evans, D. S. and Lundin, R. (1979) 'An observed relation between magnetic field aligned electric fields and downward electron energy fluxes in the vicinity of auroral forms', Journal of Geophysical Research, 84(A2), pp. 457-461. doi: 10.1029/JA084iA02p00457.

Marklund, G. T., Karlsson, T., Blomberg, L. G., Lindqvist, P.-A., Fälthammar, C.-G., Johnson, M. L., Murphree, J. S., Andersson, L., Eliasson, L., Opgenoorth, H. J. and Zanetti, L. J. (1998) 'Observations of the electric field fine structure associated with the westward traveling surge and large-scale auroral spirals', Journal of Geophysical Research, 103(A3), pp. 4125-4144. doi: 10.1029/97JA00558. 
Marklund, G. T., Sadeghi, S., Li, B., Amm, O., Cumnock, J. A., Zhang, Y., Nilsson, H., Masson, A., Karlsson, T., Lindqvist, P. A., Fazakerley, A., Lucek, E. and Pickett, J. (2012) 'Cluster multipoint study of the acceleration potential pattern and electrodynamics of an auroral surge and its associated horn arc', Journal of Geophysical Research: Space Physics, 117(10), A10223. doi: 10.1029/2012JA018046.

McPherron, R. L., Russell, C. T. and Aubry, M. P. (1973) 'Satellite studies of magnetospheric substorms on August 15, 1968: 9. Phenomenological model for substorms', Journal of Geophysical Research, 78(16), pp. 3131-3149. doi: 10.1029/JA078i016p03131.

Nishida, a. and Nagayama, N. (1973) 'Synoptic survey for the neutral line in the magnetotail during the substorm expansion phase', Journal of Geophysical Research, 78(19), pp. 37823798. doi: 10.1029/JA078i019p03782.

Nishimura, Y., Lyons, L., Zou, S., Angelopoulos, V., Mende, S., Sciences, O., Angeles, L., Physics, P., Sciences, O., Sciences, S. and Arbor, A. (2010) 'Substorm triggering by new plasma intrusion : THEMIS all-sky imager observations', Journal of Geophysical Research, 115, A07222. doi: 10.1029/2009JA015166.

Oguti, T. (1974) 'Rotational deformations and related drift motions of auroral arcs', Journal of Geophysical Research, 79(25), pp. 3861-3865. doi: 10.1029/JA079i025p03861.

Opgenoorth, H. J., Pellinen, R. J., Baumjohann, W., Nielsen, E., Marklund, G. and Eliasson, L. (1983) 'Three-dimensional current flow and particle precipitation in a westward travelling surge (observed during the Barium-Geos Rocket Experiment)', Journal of Geophysical Research, 88(A4), pp. 3138-3152. doi: 10.1029/JA088iA04p03138.

Siscoe, G. L., Crooker, N. U., Erickson, G. M., Sonnerup, B. U., Ouml, Siebert, K. D., Weimer, D. R., White, W. W. and Maynard, N. C. (2000) 'Global geometry of magnetospheric currents inferred from MHD simulations', Magnetospheric Current Systems, 118, pp. 41-52. doi: 10.1029/GM118p0041.

Song, Y. and Lysak, R. L. (2001) 'The physics in the auroral dynamo regions and auroral particle acceleration', Physics and Chemistry of the Earth, Part C: Solar, Terrestrial \& Planetary Science, 26(1-3), pp. 33-42. doi: 10.1016/S1464-1917(00)00087-8.

Tanaka, T. (1995) 'Generation mechanisms for magnetosphere-ionosphere current systems deduced from a three-dimensional MHD simulation of the solar wind-magnetosphereionosphere coupling processes', Journal of Geophysical Research, 100(A7), pp. 12057- 
12074. doi: 10.1029/95JA00419.

Tanaka, T. (2007) 'Magnetosphere-ionosphere convection as a compound system', Space Science Reviews. doi: 10.1007/s11214-007-9168-4.

Tanaka, T. (2015) ‘Substorm Auroral Dynamics Reproduced by Advanced Global Magnetosphere-Ionosphere (M-I) Coupling Simulation', in Auroral Dynamics and Space Weather. Hoboken, NJ: John Wiley \& Sons, Inc, pp. 177-190. doi: 10.1002/9781118978719.ch13.

Tanaka, T., Watanabe, M., Den, M., Fujita, S., Ebihara, Y., Kikuchi, T., Hashimoto, K. K. and Kataoka, R. (2016) 'Generation of field-aligned current (FAC) and convection through the formation of pressure regimes: Correction for the concept of Dungey's convection', Journal of Geophysical Research: Space Physics, 121(9), pp. 8695-8711. doi: 10.1002/2016JA022822.

Weimer, D. R., Craven, J. D., Frank, L. a., Hanson, W. B., Maynard, N. C., Hoffman, R. a. and Slavin, J. A. (1994) 'Satellite measurements through the center of a substorm surge', Journal of Geophysical Research, 99(A12), pp. 23639-23649. doi: 10.1029/94JA01976.

Weimer, D. R., Gurnett, D. A., Goertz, C. K., Menietti, J. D., Burch, J. L. and Sugiura, M. (1987) 'The current-voltage relationship in auroral current sheets', Journal of Geophysical Research, 92(A1), pp. 187-194. doi: 10.1029/JA092iA01p00187.

Yang, J. et al., 2012. Large-scale current systems and ground magnetic disturbance during deep substorm injections. Journal of Geophysical Research: Space Physics, 117(A4), A04223. doi: 10.1029/2011JA017415. 